\title{
Isotope fractionation in silicate melts
}

ARISING FRom G. Dominguez, G. Wilkins \& M. H. Thiemens Nature 473, 70-73 (2011)

Experiments show that temperature gradients in silicate melts lead to isotope fractionation, where the heavier isotopes concentrate in cold regions and light isotopes concentrate in hot regions ${ }^{1-4}$. Dominguez et al..$^{5}$ present a phenomenological model based on quantum effects that provides a good fit to these experimental results, and argue that "consideration of the quantum mechanical zero-point energy of diffusing species is essential for understanding diffusion at the isotopic level". However, we point out that the zero-point energy required to fit their model to experimental results is unphysically large, and that isotopic fractionation similar to that observed in silicate melts is found in systems where quantum effects are absent. Therefore, the conclusion that quantum effects underlie isotope fractionation in silicate melts with temperature gradients is not justified.

To fit experimental data, the Dominguez et al. ${ }^{5}$ model requires a zero-point energy (ZPE) for ${ }^{26} \mathrm{Mg}$ of $\sim 0.4 \mathrm{eV}$. The atomic motion giving rise to the $\mathrm{ZPE}$ is vibrational, and can be modelled by a harmonic oscillator for which ZPE $=(1 / 2) h v$, where $h$ is Planck's constant and $v$ is the vibrational frequency. (Here for convenience we consider $\tilde{v}=v / c$, where $c$ is the velocity of light.) The value $\mathrm{ZPE} \approx 0.4 \mathrm{eV}$ corresponds to $\tilde{v} \approx 6,500 \mathrm{~cm}^{-1}$, which is much larger than the highest vibrational frequencies $\left(\sim 1,300 \mathrm{~cm}^{-1}\right)$ observed in anhydrous silicate melts ${ }^{6}$. In fact, $\tilde{v} \approx 6,500 \mathrm{~cm}^{-1}$ is larger than the vibrational frequency in any material whatsoever (the highest vibrational frequency we are aware of is that for $\mathrm{H}_{2}$, where $\left.\tilde{v} \approx 4,395 \mathrm{~cm}^{-1}\right)^{7}$. Thus a ZPE of $\sim 0.4 \mathrm{eV}$ is not physically relevant.

The unphysically large ZPE in the model of Dominguez et al..$^{5}$ leads to predictions of relative diffusivities of isotopes that are in poor agreement with experiments. For example, their model (equations (11) and (12), and $\left.\mathrm{ZPE}\left({ }^{26} \mathrm{Mg}\right)=0.4 \mathrm{eV}\right)$ predicts $D\left({ }^{24} \mathrm{Mg}\right) / D\left({ }^{26} \mathrm{Mg}\right)=1.13$ at $1,500 \mathrm{~K}$. In contrast, experiments on silicate melts find $D\left({ }^{24} \mathrm{Mg}\right) /$ $D\left({ }^{26} \mathrm{Mg}\right)=1.004$ (ref. 2). Thus, the Dominguez et al. model predicts an isotope effect for relative diffusivities that is more than 30 times larger than found experimentally (13\% versus $0.4 \%)$.

Finally, we note that isotope fractionation in temperature gradients occurs in systems where quantum effects are not relevant; this implies that quantum effects are not a necessary condition for isotope fractionation to occur (whereas they are a necessary condition in the Dominguez et al. ${ }^{5}$ model). For example, significant fractionation of isotopes is seen in gases held in a temperature gradient ${ }^{8-10}$. In gases, quantum ZPE (arising from confinement) plays no role because molecules typically are far apart. Thermal fractionation of isotopes is also observed in molecular dynamics simulations of condensed phase systems ${ }^{11}$ based on classical mechanics - these simulations ignore quantum effects, and in contrast to the model of Dominguez et al..$^{5}$ include no phenomenological considerations. In both of these cases, heavier isotopes concentrate in cold regions and light isotopes concentrate in hot regions, consistent with experimental observations on silicate melts and all other condensed phase systems that have been studied. This effect is understood theoretically in terms of classical mechanics ${ }^{12}$, and quantitative agreement is obtained between this theory and experiment ${ }^{13}$.

Daniel J. Lacks ${ }^{1}$, James A. Van Orman ${ }^{2}$ \& Charles E. Lesher ${ }^{3}$

${ }^{1}$ Department of Chemical Engineering, Case Western Reserve University, Cleveland, Ohio 44106, USA.

email: daniel.lacks@case.edu

${ }^{2}$ Department of Geological Sciences, Case Western Reserve University,

Cleveland, Ohio 44106, USA.

${ }^{3}$ Department of Geology, University of California, Davis, California 95616 , USA.

\section{Received 13 May; accepted 11 November 2011.}

1. Kyser,T. K., Lesher, C. E. \& Walker, D. The effects of liquid immiscibility and thermal diffusion on oxygen isotopes in silicate liquids. Contrib. Mineral. Petrol. 133, 373-381 (1998).

2. Richter, F.M. Watson, E. B. Mendybaev, R. A., Teng,F.-Z. \& Janney, P. E. Magnesium isotope fractionation in silicate melts by chemical and thermal diffusion. Geochim. Cosmochim. Acta 72, 206-220 (2008).

3. Richter, F. M. et al. Isotopic fractionation of the major elements of molten basalt by chemical and thermal diffusion. Geochim. Cosmochim. Acta 73, 4250-4263 (2009).

4. Huang, F. et al. Isotope fractionation in silicate melts by thermal diffusion. Nature 464, 396-400 (2010)

5. Dominguez, G., Wilkins, G. \& Thiemens, M. H. The Soret effect and isotopic fractionation in high-temperature silicate melts. Nature 473, 70-73 (2011).

6. McMillan, P. F., Wolf, G. H. \& Poe, B. T. Vibrational spectroscopy of silicate liquids and glasses. Chem. Geol. 96, 351-366 (1992).

7. Karplus, M. \& Porter, R. N. Atoms and Molecules: An Introduction for Students of Physical Chemistry 369 (Benjamin-Cummings, Menlo Park, California, 1970).

8. Nier, A. O. The concentration of carbon 13 by thermal diffusion. Phys. Rev. 57, 30-34 (1940).

9. Watson, W. W. Thermal separation of isotopes. Phys. Rev. 57, 899-902 (1940).

10. Grachev, A. M. \& Severinghaus, J. P. Laboratory determination of thermal diffusion constants for ${ }^{29} \mathrm{~N}_{2} / 28 \mathrm{~N}_{2}$ in air at temperatures from -60 to $0{ }^{\circ} \mathrm{C}$ for reconstruction of magnitudes of abrupt climate changes using the ice core fossil-air paleothermometer. Geochim. Cosmochim. Acta 67, 345-360 (2003).

11. Reith, D. \& Müller-Plathe, F. On the nature of thermal diffusion in binary Lennard Jones liquids. J. Chem. Phys. 112, 2436-2443 (2000).

12. Furry, W. H., Jones, R. C. \& Onsager, L. On the theory of isotope separation by thermal diffusion. Phys. Rev. 55, 1083-1095 (1939).

13. Stier, L. G. The coefficients of thermal diffusion of neon and argon and their variation with temperature. Phys. Rev. 62, 548-551 (1942).

Author Contributions All authors contributed to the analysis and the writing of the paper.

Competing Financial Interests Declared none.

doi:10.1038/nature10764

\section{Dominguez et al. reply}

REPLYING To D. J. Lacks, J. A. Van Orman \& C. E. Lesher Nature 482, http://dx.doi.org/10.1038/nature10764 (2012)

Lacks et al. ${ }^{1}$ argue that our model of isotopic fractionation in thermal gradients in silicate melts ${ }^{2}$ does not agree with measurements of the ratio of diffusivities seen in silicate melts. This statement is based on an over-interpretation of our model into non-steady-state applications, such as chemical fractionation, because the model we presented treats the quantized energy levels of the transition state as being equal to each other (the partition function $Z(\mathrm{TS})=1$ ). This was warranted, as our main interest was in finding the steady-state solution to isotopic fractionation in a closed system (which is insensitive to the transition state). The potential importance of the transition state in determining 
the ratios of diffusivities of He isotopes in a geologic system has previously been noted ${ }^{3}$, and future work will need to clarify the importance of the transition state for kinetic isotopic fractionation in silicate systems, particularly the ratio of diffusivities.

Lacks et al. ${ }^{1}$ point out that isotopic fractionation due to temperature gradients in the gas phase have been observed (for example, ref. 4), but they do not provide proof that classical mechanics quantitatively explains these observations. Furthermore, Lacks et al. ${ }^{1}$ (and references therein) provide no evidence that molecular dynamics simulations reproduce the isotopic fractionations observed in real gas systems, much less high-temperature condensed phases. A full understanding of isotopic fractionation for complex (diatomic, polyatomic) species, even in the gas phase, is likely to require quantum mechanics because of the involvement of quantized vibrations ${ }^{5}$.

No evidence is presented by Lacks et al. ${ }^{1}$ that molecular dynamics simulations, based on Lennard-Jones interactions, are capable of reproducing the isotopic fractionations of elements and of capturing the strong potential energy interactions that characterize silicate melts and other systems where diffusion is a strong function of temperature. Because of the magnitude of the activation energies involved, only a very small fraction $(f)$ of all particles acquire enough energy to overcome activation energy barriers: $f \approx \exp -\left(-E_{\mathrm{a}} / k_{\mathrm{B}} T\right)$. Here $E_{\mathrm{a}}$ is activation energy, $k_{\mathrm{B}}$ Boltzmann's constant, and $T$ temperature. For typical activation energies of $2-3 \mathrm{eV}$, this fraction is rather small $\left(\sim 10^{-8}\right)$, thus making it difficult for molecular dynamics simulations, which typically employ $\sim 10^{3}$ particles, to realistically capture the thermodynamics associated with isotopic fractionation in a highly interacting condensed matter system. We suggest caution in interpreting simple binary mixture molecular dynamics simulations that rely on 'unphysical' means of implementing heat transport ${ }^{6}$.

Last, Lacks et al. ${ }^{1}$ point out that the vibrational frequencies needed to explain the steady-state fractionations are physically unrealistic. We note that this frequency may or may not correspond to a physical frequency, as the initial reactant state consists of three independent vibrational components that we, for the sake of clarity and simplicity, incorporated into one effective frequency (thus the effective frequency would be higher than each of the independent components) ${ }^{7}$. The infrared vibrational features that are observed in natural systems are sensitive only to differences in energy levels, not the zero-point energy. We also note that, as infrared vibrational features in a silicate melt are overwhelmingly dominated by modes associated with $\mathrm{Si}-\mathrm{O}$ stretching and bending, it is unclear whether Raman or vibrational infrared spectra include features associated with diffusing interstitial species such as $\mathrm{Mg}$, Fe and $\mathrm{Ca}$.

In short, Lacks et al. ${ }^{1}$ have provided no new data or physical model that quantitatively explains the empirical observations of steady-state isotopic fractionation in silicate melts. Furthermore, over 50 years of work in isotope effects in physical chemistry and isotope geochemistry support the role of quantum mechanics in isotopic fractionation processes.

\section{G. Dominguez ${ }^{1,2}$, G. Wilkins ${ }^{3}$ \& M. H. Thiemens ${ }^{2}$}

${ }^{1}$ California State University, San Marcos, Department of Physics, California 92096, USA.

email: gdominguez@csusm.edu

${ }^{2}$ University of California, San Diego, Department of Chemistry and

Biochemistry, California 92093, USA.

3University of California, San Diego, Department of Mathematics, California 92093, USA.

1. Lacks, D. J., Van Orman, J. A. \& Lesher, C. E. Isotope fractionation in silicate melts. Nature 482, http://dx.doi.org/10.1038/nature10764 (this issue).

2. Dominguez, G., Wilkins, G. \& Thiemens, M. On the Soret effect and isotopic fractionation in high-temperature silicate melts. Nature 473, 70-73 (2011).

3. Trull, T. W. \& Kurz, M. D. Isotopic fractionation accompanying helium diffusion in basaltic glass. J. Mol. Struct. 485-486, 555-567 (1999).

4. Grachev, A. M. \& Severinghaus, J. P. Laboratory determination of thermal diffusion constants for ${ }^{29} \mathrm{~N}_{2} /{ }^{28} \mathrm{~N}_{2}$ in air at temperatures from -60 to $0{ }^{\circ} \mathrm{C}$ for reconstruction of magnitudes of abrupt climate changes using the ice core fossil-air paleothermometer. Geochim. Cosmochim. Acta 67, 345-360 (2003).

5. Saxena, S. C. \& Mathur, B. P. Thermal diffusion in isotopic gas mixtures and intermolecular forces. Rev. Mod. Phys. 38, 380-390 (1966).

6. Reith, D. \& Florian, M.-P. On the nature of thermal diffusion in binary LennardJones liquids. J. Chem. Phys. 112, 2436-2453 (2000).

7. Vineyard, G. H. Frequency factors and isotope effects in solid state rate processes. J. Phys. Chem. Solids 3, 121-127 (1957).

doi:10.1038/nature10765 